\title{
Hierarchical structures in cold-drawn pearlitic steel wire
}

\author{
Zhang, Xiaodan; Godfrey, Andrew ; Hansen, Niels; Huang, Xiaoxu
}

Published in:

Acta Materialia

Link to article, DOI:

10.1016/j.actamat.2013.04.057

Publication date:

2013

Document Version

Early version, also known as pre-print

Link back to DTU Orbit

Citation (APA):

Zhang, X., Godfrey, A., Hansen, N., \& Huang, X. (2013). Hierarchical structures in cold-drawn pearlitic steel wire. Acta Materialia, 61(13), 4898-4909. https://doi.org/10.1016/j.actamat.2013.04.057

\section{General rights}

Copyright and moral rights for the publications made accessible in the public portal are retained by the authors and/or other copyright owners and it is a condition of accessing publications that users recognise and abide by the legal requirements associated with these rights.

- Users may download and print one copy of any publication from the public portal for the purpose of private study or research.

- You may not further distribute the material or use it for any profit-making activity or commercial gain

- You may freely distribute the URL identifying the publication in the public portal

If you believe that this document breaches copyright please contact us providing details, and we will remove access to the work immediately and investigate your claim. 


\title{
Hierarchical structures in cold-drawn pearlitic steel wire
}

\author{
Xiaodan Zhang ${ }^{1,2}$, Andrew Godfrey ${ }^{1}$, Niels Hansen ${ }^{2}$ and Xiaoxu Huang ${ }^{2}$ \\ ${ }^{1}$ Advanced Materials Laboratory, Department of Materials Science and Engineering, \\ Tsinghua University, 100084, PR China \\ 2 Danish-Chinese Center for Nanometals, Materials Science and Advanced \\ Characterization Section-MAC, Department of Wind Energy, Risø Campus, \\ Technical University of Denmark, DK-4000 Roskilde, Denmark
}

Key words: Hierarchical structures; Pearlitic steel wire; High resolution electron backscattered diffraction; Nanobeam diffraction; Ferrite crystallographic orientation; Dislocation structures; Strengthening mechanisms; Strengthening parameters;

\section{Abstract}

The microstructure and crystallography of drawn pearlitic steel wires have been quantified by a number of electron microscopical techniques including scanning electron microscopy, transmission electron microscopy, high resolution electron backscattered diffraction and nanobeam diffraction, with focus on the change in the structure and crystallography when a randomly oriented cementite structure in a patented wire during wire drawing is transformed into a lamellar structure parallel to the drawing axis. Changes in the interlamellar spacing and in the misorientation angles along and across the ferrite lamellae show significant through-diameter variations in wires drawn to large strain $\geqslant 1.5$. The structural evolution is hierarchical as the structural variations have their causes in a different macroscopic orientation of the cementite colonies in the initial (patented) structure with respect to the wire axis. The through-diameter variations subdivide the lamellar structure into two distinctly different types: one is called A_A with a smaller interlamellar spacing (ILS) and smaller dislocation density and the other called A_BC. During drawing the thickness of the ferrite and cementite are reduced to $20 \mathrm{~nm}$ and $2 \mathrm{~nm}$ respectively and in parallel high angle boundaries form in the A_BC structure parallel to the cementite lamellae. The structural and crystallographic analyses suggest that boundary strengthening and dislocation strengthening are important mechanisms in the cold-drawn wires. However, differences in structural parameters in the A_A and A_BC structures may affect the relative contributions of the two mechanisms to the total flow stress.

\section{Introduction}

Cold-drawn pearlitic steel wire is nanostructured and the flow stress at room temperature can reach values above $6 \mathrm{GPa}$ [1]. The wire is also characteristic by showing no yield point and it has sufficient ductility to allow shaping of industrial components. It follows that the wire is a unique nanostructured metallic material and that a characterization and analysis of structure, strengthening mechanisms and strength-structure relationships are of both scientific and applied interest. In a recent

Please cite this paper as following

X Zhang, A Godfrey, N Hansen, X Huang. Hierarchical structures in cold-drawn pearlitic steel wire. Acta Materialia 2013;61:4898-4909. 
paper [2] such an analysis has been based on a structural characterization by transmission electron microscopy (TEM) and tensile testing of wires drawn to a true strain of 3.68 and a flow stress of $3.4 \mathrm{GPa}$. Based on this analysis it has been suggested that major strengthening mechanisms are boundary strengthening, dislocation strengthening and solid solution hardening due to carbon enrichment of the ferrite phase and that these strength contributions from these mechanisms are linearly additive. This analysis supplements previous analysis during the last 50 years of strengthening mechanisms and properties based on microscopy, mechanical testing and analysis of concentration and distribution of carbon atoms in the ferrite phase by atom probe techniques [3-9].

Previous work has shown that a significant structural change takes place during drawing from the original patented wire having a diameter of a few millimeters to the final wire with the diameter of a few tens of micrometers. These microstructural changes are primarily caused by a reorientation of the cementite phase with a typical lamellar structure of alternating cementite and ferrite. However the pearlite in the undeformed wire is in colonies with different macroscopic orientations of the lamellae with respect to the drawing direction (Fig. 1). During wire drawing from small to medium strain the structure evolves into a lamellar/fibrous structure where the cementite lamellae are almost parallel to the drawing direction. This reorientation is complete at a strain of 1.5 [10-12] and it involves processes such as breakup of cementite [10, 13], curling [14] and shear banding [15]. As a result of the deformation, the microstructure evolves from being heterogeneous at low and medium strains to an apparent homogeneous structure at a large strain which continues to refine as the strain is increased. The degree of reorientation depends on the original angle between the cementite lamellae and the drawing direction. The larger the angle, the larger the reorientation and structural change. This evolution suggests that although the lamellar structure at a large strain appears homogeneous on the macroscopic scale it is a hierarchical structure with characteristics which may relate to the original orientations of the cementite lamellae $[16,17]$. As a result the structural parameters in the drawn wire determined as average values may show significant through-diameter variations. This evolution is investigated in the present study on the basis of a detailed analysis of ( $\mathrm{i}$ ) the spacing between the cementite lamellae (the interlamellar spacing (ILS)), (ii) the dislocation structure in the ferrite lamellae and (iii) the orientation change along and across the ferrite lamellae which are related to the original structure of the patented wire. The analysis of structural parameters forms the basis for a discussion of strengthening mechanisms and strength-structure relationships of the drawn wire also taking into account a carbon enrichment of the ferrite lamellae due to the decomposition of the cementite.

\section{Experimental}

Five samples at five cold-drawing strains of a high-strength near-eutectoid composition steel with a carbon content of $0.8 \mathrm{wt} \%$ supplied by NV Bekaert SA (Zwevegem, Belgium) Technology Center Laboratory were investigated, representing

Please cite this paper as following

X Zhang, A Godfrey, N Hansen, X Huang. Hierarchical structures in cold-drawn pearlitic steel wire. Acta Materialia 2013;61:4898-4909. 
intermediate steps of the manufacturing process from the as-patented wire $(1.26 \mathrm{~mm})$ to final drawn wire $(0.2 \mathrm{~mm})$. The sample details are listed in Table 1 .

Standard grinding and electro-polishing (10\% perchloric acid in ethanol) procedures were used for the preparation of specimens for investigations by scanning electron microscopy (SEM), high resolution electron backscattered diffraction (HREBSD) and by TEM. The wires were examined in the longitudinal section. For the SEM observations specimens were additionally etched in 4\% Nital. HREBSD was used to measure the crystallographic orientation change, as in the analysis of a graded nanostructured steel [18]. The step sizes for HREBSD were 40nm and 20nm. Dislocation configurations and density were examined using a JEOL 2000FX TEM at $200 \mathrm{kV}$ and a JEOL 3000 FEG-TEM at 300kV. Quantitative orientation measurements based on nano-beam diffraction spot indexing and manual fitting of simulated spot and Kikuchi patterns with measured ones have been applied [19], giving an angular resolution of misorientation angle of approximately $0.5^{\circ}$. The distance between neighboring measurement points along the ferrite lamellae corresponds to the average thickness of ferrite lamellae (F) and the distance between neighboring measurement points across the ferrite lamellae corresponds to the average interlamellar spacing (ILS) (see Table 2).

\section{Results}

\subsection{Initial Structure}

In the initial as-patented condition the pearlite structure is composed of alternating ferrite and cementite lamellae and each original austenite grain transforms into several pearlite colonies. The average size of a pearlite colony is around $6 \mu \mathrm{m}$ and the size range is from $3.3 \mu \mathrm{m}$ to about $12 \mu \mathrm{m}$. This structure relates to the patenting process used in the production. The typical pearlite structure shows no preferred macroscopic orientation of the cementite plates to the drawing direction (see Fig. 1). The demonstration of ferrite misorientation angle measurement in and across lamellae is shown in Fig 2. The misorientation angles to the starting point and the angle between neighboring points along and across ferrite lamellae are about $0.5^{\circ}$. These misorientations are close to the angular resolution of the nanobeam diffraction (NBD) technique, illustrating that the misorientation angles in a pearlite colony are small.

\subsection{Cementite and ferrite alignment during drawing}

Lamellar pearlite is formed by the isothermal decomposition of eutectoid austenite. Several differently oriented pearlite colonies are formed within one former austenite grain. Here the pearlite colony is defined as an area with a similar ferrite crystallographic orientation, continuous/discontinuous cementite lamellae and surrounded by tiny cementite particles which are easy to differentiate in the TEM at low and medium strains $(0<\varepsilon \leqslant 1.51)$ [20]. The macroscopic evolution of the structural alignment has been investigated in our previous study [10] using the cementite plates as markers. In the longitudinal section the pearlite colonies were classified into three macroscopic orientation classes, based on the angle between the length direction of the cementite plates and the wire axis $\left(0^{\circ}-30^{\circ}, 30^{\circ}-60^{\circ}\right.$ and $60^{\circ}$

Please cite this paper as following

X Zhang, A Godfrey, N Hansen, X Huang. Hierarchical structures in cold-drawn pearlitic steel wire. Acta Materialia 2013;61:4898-4909. 
- 90 ; hereafter called A, B, and C structure respectively), and into five length classes $(0-1 \mu \mathrm{m}, 1-2 \mu \mathrm{m}, 2-3 \mu \mathrm{m}, 3-4 \mu \mathrm{m}$, and $>4 \mu \mathrm{m})$. The results have clearly shown that the ferrite/cementite lamellae rotated towards and are lengthened and thinned along the wire axis with increasing strain, in agreement with previous observations $[16,21]$. At strains higher than 1.51 the ferrite/cementite lamellae become aligned parallel to the drawing direction with an angular spread of $30^{\circ}(97 \%$ at strain 1.5 , $100 \%$ at strain 3.7) [10]. Additionally TEM measurements of the average thickness of ferrite and cementite lamellae show that they reduce in correspondence with the decrease in the wire diameter except at a high strain where an additional thinning of cementite is observed [2].

The deformation of the pearlite structure is strongly related to plastic deformation in the ferrite including curling which is observed in the cross section [4, 12-14, 16, 22]. Deformation of the hard phase (cementite) is also observed as coarse slip steps, S-bands and cracks across the cementite. The slip traces are parallel to $\{110\}_{\alpha-\mathrm{Fe}}$ or $\{112\}_{\alpha-F e}$ plane traces representing the largest Schmid factors [10]. Several previous investigations have reported that $\{110\}<11-1>_{\mathrm{Fe} 3 \mathrm{C}}$, and slip on $\{100\}_{\mathrm{Fe} 3 \mathrm{C}}$ can be activated in wire drawing $[23,24]$, and slip on $(12-1)_{\mathrm{Fe} 3 \mathrm{C}} /(-110)_{a}-\mathrm{Fe}$ has also observed in $10 \%$ cold-rolled pearlitic steel sheets [25]. These results indicate that slip initially takes place in the ferrite lamellae and may transfer through the structure into the cementite lamellae. Taking into account these observations five different paths for changing the macroscopic orientation and thickness of the ferrite lamellae, have been suggested, as illustrated in Fig. 3. In this figure, A, shows a favorable orientation of cementite plates in relation to the drawing axis since the plates will not act as a strong barriers for dislocation slip in the ferrite phase which with a good approximation will deform uniformly. In contrast when the cementite plates are strongly inclined or perpendicular to the wire axis as in $\mathrm{B}$ and $\mathrm{C}$ the applied deformation causes a rigid body rotation, resulting in twisting and breakup of cementite platelets. At high strains all the ferrite lamellae become aligned close to the drawing direction and with increasing strain the ferrite lamellae are lengthened and thinned as observed in the longitudinal section and the transverse section, respectively. This progressive evolution of the microstructure is in agreement with previous observations $[10,13,16]$ and calculations [12].

3.3 Structural classification

Fig. 3 illustrates a hierarchical evolution of the deformation structure with increasing strain. This evolution takes place along different deformation paths which may lead to through-diameter variations in structural morphology and crystallography. Based on Fig. 3 three different structural types may be identifiable in the drawn wire:

A_A: A structure which represents the part of the deformation structure which has its origin in A colonies in the patented wire;

A_B and A_C: structures which represent the parts of the deformation structure which have their origins in $\mathrm{B}$ and $\mathrm{C}$ colonies, respectively in the patented wire;

At low to medium strains A_A structures have not undergone significant changes

Please cite this paper as following

X Zhang, A Godfrey, N Hansen, X Huang. Hierarchical structures in cold-drawn pearlitic steel wire. Acta Materialia 2013;61:4898-4909. 
when compared to the original A structure and A_B and A_C structures can be identified due to their content of S-bands, shear bands etc. However at a strain of about 1.5 a clear distinction between A_B and A_C structures is not longer possible and they are classified together as an A_BC structure. The percentage of A_A and A_BC structures in the cold-drawn wire is in Table 3 compared to the area percentage of $\mathrm{A}$ and $\mathrm{B} / \mathrm{C}$ structures in the patented wire. A good agreement can be observed illustrating the hierarchical structural evolution during wire drawing up to a strain of 1.51 .

An application of morphological differences as a criteria for separating between A_A and A_BC structures is however not applicable at large strains as the structures appear as a deformed structure with uniform morphology. However it has been found by measuring misorientation angles across and along the ferrite lamellae that the apparent uniform structure is subdivided into volume elements with very different crystallographic characteristics. An example is shown in Fig. 4 where Fig. 4c shows that part of the volume is subdivided by lamellar boundaries with an angle which can reach $40^{\circ}$, i.e., high angle boundaries. In contrast other areas only shows low angle boundaries $\left(<15^{\circ}\right)$. This significant difference suggests a structural subdivision into A_A and A_BC structures where the A_A structure is undergoing almost uniform deformation, and only subdivides by the formation of low angle boundaries. A misorientation angle profile measured along the ferrite lamellae is shown in Fig. $4 \mathrm{~d}$ for the A_A structure and in Fig. 4e for the A_BC structure. The misorientation angles are small however significantly larger in the A_BC structure than in the A_A structure.

Based on the analysis above of morphology and crystallography the following guidelines are used for the characterization of the lamellar structure in the strain range from 1.51 to 3.68 .

- Misorientation angles across ferrite lamellae are small in A_A structures, less than $3^{\circ}$ and large in A_BC structures, up to $40^{\circ}$.

- Misorientation angles along ferrite lamellae are small in A_A structures, less than a few degrees and large in A_BC structures, up to $7^{\circ}$.

\subsection{Structural parameters}

The subdivision of the lamellar structure into regions classified as A_A and A_BC regions will affect the structural parameters which may show through-diameter variation in the cold-drawn wire. The criteria suggested in Section 3.3 form the basis for an analysis by HREBSD and TEM in order to quantify important parameters and their local variations. Examples which will be discussed in the following are: $(i)$ the interlamellar spacing (ILS), (ii) the angular changes across the ferrite lamellae and (iii) the angular changes along the ferrite lamellae and the dislocation structure in these lamellae.

\subsubsection{Interlamellar spacing (ILS)}

The average interlamellar spacing has been quantified for the initial structure (A, B and $\mathrm{C}$ orientations) and for A_A and A_BC structures in the strain range from 1.51 to

Please cite this paper as following

X Zhang, A Godfrey, N Hansen, X Huang. Hierarchical structures in cold-drawn pearlitic steel wire. Acta Materialia 2013;61:4898-4909. 
3.68. The spacing are given in Table 4 which shows a decrease in ILS with increasing strain and that the ILS in the A_A structure is significantly smaller than the ILS in the A_BC structure. The standard deviations in Table 4 however also show that there is a significant overlap of the distributions which is illustrated in Fig. 5 for $\varepsilon=2.67$ where data are reported separately for A_A and A_BC structures and in Fig. 6 where the numbers are combined. The distributions in Fig. 5 and Fig. 6 are not analyzed in detail but they illustrate that the total distribution is far from being bimodal and that the width of the distributions is comparable to a typical grain size distribution in a recrystallized metal [26].

In general the decrease in ILS with the increasing strain is assumed to follow a $\varepsilon^{-0.5}$ power law relationship where $\varepsilon$ is the true strain. However as shown in Fig 7 this relationship agrees well with the experimental finding for A_A structure (Fig. 7a) but significantly under predict the ILS for A_BC structure (Fig. 7b). However when the numbers are combined (Fig. 7c) and taking into account the fairly large standard deviations it appears that an analysis based on average values and on a $\varepsilon^{-0.5}$ power law relationship gives a reasonable description of the structural refinement of the lamellar structure at increasing strain.

\subsubsection{Angular change across the ferrite lamellae}

The analysis by HREBSD shows that one part of the structure of the drawn wire is characterized by small misorientation angles while other regions show very large misorientation angles when measured perpendicular to the lamellar boundaries. The TEM observations shown in Fig. 8 support the HREBSD findings. Typically in a small angle region the misorientation angle is smaller than $3^{\circ}$ with a tendency to increase as the strain is increased from 2.67 to 3.68 (Fig. 8). For a large angle region the misorientation angle across neighboring lamellae can reach $40^{\circ}$ (Fig. 9). The structure in Fig. 8 can therefore be classified as A_A structures while the structure in Fig. 9 is classified as an A_BC structure. The misorientation angle across the ferrite lamellae determined by HREBSD and TEM as a function of strain is plotted together in Fig. 10. The orientation changes observed in the initial structure (A, B and C) are comparable and the misorientation angle between neighboring points are less than $1^{\circ}$. For small and medium strains large misorientation angles characterize $\mathrm{B}$ and $\mathrm{C}$ structures and to a less extent the A structure, reflecting the different degree of cementite reorientation in this strain range. Also at a strain larger than 1.5 this difference is observed indicating that although the morphology in A_A and A_BC structures appear uniform the large angular spread introduced at low and medium strains in the A_BC structure persists to the largest strain.

\subsubsection{Angular changes along the ferrite lamellae}

The analysis by HREBSD and TEM shows that the changes in misorientation angles along the ferrite lamellae are relatively small reflecting the presence of structural defects such as dislocations. Figure 11 shows the microstructure and ferrite misorientation change along the lamellae in an A_A structure at a strain of 3.68. One $\mathrm{LAB}$ with a misorientation angle of $1.3^{\circ}$ has formed in the ferrite lamella. Compared

Please cite this paper as following

X Zhang, A Godfrey, N Hansen, X Huang. Hierarchical structures in cold-drawn pearlitic steel wire. Acta Materialia 2013;61:4898-4909. 
with the LABs in the A_A structure, the number and misorientation angles of LABs in the A_BC structure with an approximately similar lamellar thickness are larger. As shown in Fig. 12, five LABs with misorientation angles larger than $2^{\circ}$ (ranging from $2.4^{\circ}$ to $7.2^{\circ}$ ) in the ferrite lamellae with a thickness around $12 \mathrm{~nm}$, have formed over a length of $250 \mathrm{~nm}$. The misorientation profile to the first point also shows an accumulative characteristic. In supplement to the dislocations stored in boundaries threading dislocations are present with a density which at high strain can reach $1 \times 10^{16}$ $\mathrm{m}^{-2}[2]$.

Fig. 13 summarizes the ferrite orientation change along the lamellae as a function of strain $(\geqslant 1.51)$. In Figure 13 TEM and HREBSD data are combined but as the distances between measurements are different in the two cases, the measurements are normalized by the distance between neighboring points. The clear trend is that the value and spread of normalized misorientation angles along the ferrite lamellae increases with the strain for all structures, and is significantly larger in the A_BC structure than in the A_A structure. This may be related to the strain path difference at low and medium strains.

\section{Discussion}

\subsection{Hierarchical structural evolution}

The lamellar structure giving the strength to cold-drawn pearlitic steel wire originates from a patented structure composed of pearlite colonies representing different macroscopic orientations of the cementite platelets with respect to the wire axis [10, 16]. These cementite platelets reorient to the wire axis and observations $[10,16]$ and calculations [12] show that this reorientation is almost complete at a true strain of about 1.5. The lamellar structure is composed of cementite and ferrite lamellae which have undergone pronounced plastic deformation in the low and medium strain range characterized by, for example: deformation and fracture of the cementite [3-5, 10, 22], localized shearing of the cementite and curling of the ferrite [10, 14-16, 22]. These processes introduce dislocations into the ferrite phase and cause crystallographic rotation of the ferrite lattice. As to the dislocation density, an increase with increasing strain must be expected due to the formation of geometrically necessary dislocations in order to accommodate strain gradients caused by curling and by glide interaction between a hard cementite phase and a soft ferrite phase. These dislocations together with statistically stored dislocations will affect the misorientation angle along the ferrite lamellae which is expected to increase with increasing dislocation density in the lamellae. As to lattice rotation of the ferrite phase it leads to an evolution of a wire texture with a composition and strength which may be affected by the degree of structural reorientation taking place during wire drawing. It follows that both the formation and storage of dislocations in the ferrite lamellae and the crystallographic rotation of such lamellae may be directly related to the reorientation of the pearlite colonies. It may also be expected that these parameters will be affected by the degree of structural reorientation, i.e., with an increasing angle between the cementite platelets in the patented wire and the wire axis. It follows from such a hypothesis that

Please cite this paper as following

X Zhang, A Godfrey, N Hansen, X Huang. Hierarchical structures in cold-drawn pearlitic steel wire. Acta Materialia 2013;61:4898-4909. 
the wire structure at a large strain may show through-diameter variations reflecting that the structural evolution during drawing has followed a hierarchical pattern however along different deformation paths. This has been observed in the present study and allowed a separation into two different deformation paths:

1) From an A to A_A structure where cementite lamellae in A form an angle of $0-30^{\circ}$ with wire axis and $\mathrm{A} \_\mathrm{A}$ is characterized by relatively small changes in misorientation angle along and across the ferrite lamellae;

2) From a $B$ and $C$ to $A$ _ BC structure where cementite lamellae in $B$ or $C$ form an angle of $30-90^{\circ}$ with the wire axis and A_BC is characterized by relatively large changes in misorientation angle along and across the ferrite lamellae.

These characteristic structures will be the basis for the discussion which is related to a previous analysis of structural parameters, strengthening mechanisms and strength-structural relationships in drawn pearlitic steel wire [2]. The key structural parameters were the interlamellar spacing (ILS), the dislocation density in the ferrite lamellae and the concentration of carbon in the ferrite. Based on these parameters the strength-structure relationships were analyzed, on the assumption of boundary strengthening, dislocation strengthening and solid solution hardening. In the previous analysis the structural parameters were average values but as through-diameter structural variations build-up during wire drawing it is relevant to analyze the impact of such variations on the strengthening mechanisms and the strength-structure relationship also taking into account a carbon enrichment of the ferrite due to decomposition of the cementite. In this analysis is maintained the original three strengthening mechanisms [2] as the structural characterization does not indicate changes in the basic strengthening mechanisms. However the magnitude of the structural parameters show changes which may affect the relative contributions of the two mechanisms to the total flow stress.

\subsection{Boundary strengthening}

The strengthening due to the hard cementite lamellae has been analyzed [2] as a Hall-Petch strengthening with the flow stress being proportional to $\mathrm{k} \cdot \mathrm{d}^{-0.5}$ where $\mathrm{d}$ is the average boundary or barrier distance and $\mathrm{k}$ is a measure of the boundary strength which according to ref. 27 equals to $\tau_{\varepsilon} \gamma^{0.5}$ where $\tau_{\varepsilon}$ is the stress to operate a source at a distance of $\gamma$ from the boundary. The barrier distance taken to be proportional with the interlamellar spacing decreases with increasing strain and is assumed to follow a $\varepsilon^{-0.5}$ power law relationship where $\varepsilon$ is the true strain. This relationship may be valid for wire drawing where the initial orientation of the cementite is parallel to the wire axis and the strain path is near uniform. This condition covers the transition from $\mathrm{A}$ to A_A but may show deviations for the less uniform strain paths from B and C to A_BC. As a result it must be expected that the interlamellar spacing differs between A_A and A_BC structures and this is observed, see Table 4. It is also found that interlamellar spacing is smaller in the A_A structure than in the A_BC structure and that the $\varepsilon^{-0.5}$ power law relationship slightly over predicts the evolution of the ILS in A_A structure but under predicts the ILS for the A_BC structure. This is in accord

Please cite this paper as following

X Zhang, A Godfrey, N Hansen, X Huang. Hierarchical structures in cold-drawn pearlitic steel wire. Acta Materialia 2013;61:4898-4909. 
with phenomenological and Taylor-type micromechanical modeling [28] showing for a random distribution of cementite (as in the patented wire) that the $\varepsilon^{-0.5}$ dependency overestimates the decrease of lamellar spacing during wire drawing whereas this dependency is valid when lamellae are already aligned parallel to the wire axis as in the A colony in the patented wire.

The difference in the ILS between A_A and A_BC structures may not affect the basic boundary strengthening mechanism but it will result in a difference in the flow stress according to the Hall-Petch relationship. However based on the finding that the ILS in A_A and A_BC structures are not significantly different taken the large standard deviation into account it is considered acceptable to use the average ILS when calculating the flow stress [2]. Note also that the distribution of ILS is near normal and that the very large spacing which could have reduced $\mathrm{k}_{1}$ [29] are not present.

In parallel to the decrease in lamellar spacing during wire drawing the thickness of the cementite lamellae is reduced also according to a power law $\varepsilon^{-0.5}$ relationship [2, 30]. However at large strain the relationship appears to overestimate the thickness of the cementite which has been related [2] to a decomposition of the cementite. Thus both thinning and decomposition may reduce the resistance of the cementite boundary to dislocation glide. However, the resistance of the boundary may also increase with increasing strain as the misorientation angle measurement across the lamellae shows rotation of the ferrite lamellar around the wire axis. This rotation is not pronounced in the A_A structure but a large rotation is observed in the A_BC structure. The rotation angle can be as high as $40^{\circ}$, which means that high angle boundaries have formed almost parallel to the cementite lamellae. In summary the observation points to the boundary strengthening is an important strengthening mechanism with a contribution proportional to the inverse square root of the ILS also taking into account that the strain-induced high angle boundaries may be strengthened through interaction with carbon atoms in the ferrite from the decomposition of the cementite [2, 6-9, 31-36].

\subsection{Dislocation strengthening}

The strengthening due to dislocations has been analyzed based on an assumption of forest hardening [2] and a proportionality between the flow stress and $\rho^{0.5}$ where $\rho$ is the average dislocation density in the ferrite lamellae. The dislocations are stored: (i) as threading dislocations, (ii) at the ferrite-cementite interface and (iii) in low angle cell or subgrain boundaries. These three types of configurations are observed both in A_A and A_BC structures. It is also observed that cell/subgrain boundaries form readily in both types of structures indicating a fairly high mobility of dislocations both in $2 \mathrm{D}$ and $3 \mathrm{D}$ even if only a small volume is available in the lamellae. A cause may be the rather complex evolutionary path which characterized both A_A and A_ BC structures. This process may activate a number of different slip systems thereby increasing the dislocation mobility and interaction between glide dislocations. As a result both the density of statistically stored and geometrically necessary dislocations will increase at a given strain, but it must be expected that the dislocation density at a given strain will be higher in the A_BC structure than in the A_A structure. This has

Please cite this paper as following

X Zhang, A Godfrey, N Hansen, X Huang. Hierarchical structures in cold-drawn pearlitic steel wire. Acta Materialia 2013;61:4898-4909. 
been investigated by comparing the misorientation angle distribution when measuring in and along the ferrite lamellae. The magnitude of this angle reflects the dislocation density in the lamellae independent of their specific arrangement, showing that the dislocation density is significantly smaller in the A_A than in the A_BC structure.

In summary the observations point to the dislocation strengthening as an important strengthening mechanism with a contribution proportional to the dislocation density and that the contribution of dislocations to the flow stress will be larger in the A_BC than in the A_A structure. As for the boundary strengthening the interaction between dislocations and carbon atoms may also have to be taken into account.

4.4 Solid solution hardening

Solid solution hardening is introduced, as carbon enrichment of the ferrite is expected to lead to hardening due to the interaction between dislocations in different configurations and carbon atoms which are introduced through dissolution of cementite. Such a cementite dissolution during the plastic deformation of steels has been detected by various techniques, including Mössbauer spectrometry [33, 34, 36], internal friction [33], thermomagnetic analysis [33, 37], electron energy loss spectrometry (EELS) [32] and atom probe microscopy [1, 6-9, 35, 38]. Basically, two mechanisms have been proposed: one attributes the decomposition of cementite to a higher enthalpy of binding between carbon atoms and dislocations in ferrite as compared with the binding of carbon and iron atoms in cementite [36]; the other is based on the destabilization of the cementite due to a increase of its free energy arising from thinning of cementite lamellae and creation of slip steps during wire drawing [32]. However, which one is dominating is still in debate where it also may be taken into account the orientation measurements across ferrite lamellae in as-drawn wires showing that the complete dissolution of cementite is likely to leave behind either a high angle (A_BC regions) or a low angle (A_A regions) grain boundary. For a further identification of the mechanisms which controls the cementite dissolution, a combined analysis of changes in chemical-composition and deformation and subdivision of cementite during deformation could be a next step.

The effect of carbon in solution has not been included in the present study as it has been dealt with in many current investigations applying the atom probe technique [1, 6-9, 35]. Such studies may also include interaction between solute atoms and cell/subgrain boundaries as observed by atom probe TEM and by TEM [1]. A comparison is however difficult due to the different spatial resolution of the two techniques, whereas it must be expected that the through-diameter variation especially in the dislocation density may also affect the degree of cementite decomposition. An estimate of this effect is for future research, also encompassing structural evolution, deformation mechanisms and strength structural relationship at a very high strain to about 6 resulting on wires with a flow stress above $6 \mathrm{GPa}[1,39]$.

\section{Conclusions}

The microstructure of pearlitic steel wires cold-drawn to strains up to 3.7 have been

Please cite this paper as following

X Zhang, A Godfrey, N Hansen, X Huang. Hierarchical structures in cold-drawn pearlitic steel wire. Acta Materialia 2013;61:4898-4909. 
quantified by a number of electron microscopical techniques, with focus on the change in structure and crystallography where a randomly oriented cementite structure in a patented wire during wire drawing is transformed into a lamellar nanostructure parallel to the drawing axis. The conclusions are the following:

- Changes in ILS and in the misorientation angles along and across the ferrite lamellae show significant through-diameter variations in wires drawn to a large strain $\geqslant 1.5$. The structural evolution is hierarchical as the structural variations have their causes in a different macroscopic orientation of the cementite in the initial (patented) structure with respect to the wire axis.

- The through-diameter variations subdivide the lamellar structure into two types A_A and A_BC where the latter has a larger ILS and a higher dislocation density. The thickness of the ferrite and cementite is reduced to $20 \mathrm{~nm}$ and $2 \mathrm{~nm}$, respectively, and in parallel high angle boundaries form in the A_BC structure parallel to the cementite lamellae.

- In both A_A and A_BC structures the dislocations are as stored individual dislocations as they are stored in low angle cell or subgrain boundaries. The misorientation angles vary in the range $1-8^{\circ}$ and the boundaries are observed even in ferrite lamellae as thin as $10-20 \mathrm{~nm}$.

- The structural and crystallographic analyses suggest that the boundary strengthening with a contribution proportional to the inverse square root of the ILS and dislocation strengthening with a contribution proportional to the square root of the dislocation density are important mechanisms in the A_A and A_BC structures. However the differences in structural parameters as the boundary spacing and the dislocation density between the two structures may affect the relative contributions of the two mechanisms to the total flow stress.

\section{Acknowledgements}

The authors thank NV Bekaert SA Technology Center Laboratory (Zwevegem, Belgium) for the supply of the pearlitic steel wires used in this investigation. The authors gratefully acknowledge the support from the Danish National Research Foundation (Grant No DNRF86-5) and the National Natural Science Foundation of China (Grant No. 51261130091) to the Danish-Chinese Center for Nanometals, within which this work has been performed. The authors also thank B. Ralph for his useful comments during preparation of the manuscript.

\section{References}

[1] Li YJ, Choi P, Goto S, Borchers C, Raabe D, Kirchheim R. Acta Mater 2012;60:4005.

[2] Zhang X, Godfrey A, Huang X, Hansen N, Liu Q. Acta Mater 2011;59:3422.

[3] Embury JD, Fisher RM. Acta Metall 1966;14:147.

[4] Langford G. Metall Trans 1977;8A:861.

[5] Gil Sevillano J. J Phys III 1991;1:967.

[6] Read H G, Reynolds W T, Hono K et al. Scripta Mater 1997; 37: 1221.

[7] Hong MH, Reynolds WT, Tarui T, Hono K. Metall Mater Trans 1999;30A: 717.

Please cite this paper as following

X Zhang, A Godfrey, N Hansen, X Huang. Hierarchical structures in cold-drawn pearlitic steel wire. Acta Materialia 2013;61:4898-4909. 
[8] Hono K, Ohnuma M, Murayama M, Nishida S, Yoshie A, Takahashi T. Scripta Mater, 2001;44: 977.

[9] Takahashi J, Tarui T, Kawakami K. Ultramicroscopy 2009;109:193.

[10] Zhang X, Godfrey A, Hansen N, Huang X, Liu W, Liu Q. Mater Charac 2010; 61:65.

[11] Zhang X, Hansen N, Godfrey A, Huang X. In: Proceedings of the 33rd Risø international symposium on materials science: Nanometals - status and perspectives. Risø, Denmark: DTU; 2012. p. 407-16.

[12] Gil Sevillano J. PhD thesis, Katholieke Universiteit Leuven, Belgium; 1974.

[13] Zhang XD, Godfrey A, Liu W, Liu Q. Mater Sci Technol 2010;27:562.

[14] Hosford WF. Trans Met Soc AIME 1964; 230: 12.

[15] Aernoudt E, Van Houtte P, Leffers T. Deformation and textures of metals at large strain. Plastic deformation and fracture of materials. Ed.: Hael Mughrabi. Weinheim, Newyork; Basel; Cambridge; VCH, 1993; P 129.

[16] J. Toribio, E. Ovejero. Scripta Mater 1998;39:323.

[17] Elices M. Journal of Materials Science 2004;39:3889.

[18] Zhang X, Hansen N, Gao Y, Huang X. Acta Mater 2012;60:5933.

[19] Sugio K, Liu HH, Poulsen HF, Huang X. In: Proceedings of the 30th Risø international symposium on materials science: nanostructured metals-fundamentals to applications. Risø, Denmark: DTU; 2009. p. 337-42.

[20] Zhang XD. PhD thesis, Tsinghua University, Beijing; 2009.

[21] Zelin M. Acta Mater 2002;50:4431.

[22] Langford G. Metall Trans 1970;1:465.

[23] Karlsson B, Linden G. Mater Sci Eng 1975;17:153.

[24] Karlsson B, Linden G. Mater Sci Eng 1975;17:209.

[25] Tagashira S, Sakai K, Furuhara T, Maki T. ISIJ Int 2000;40:1149.

[26] Juul Jensen D. Scripta Metall Mater 1992;27:533.

[27] Contrell AH. The mechanical properties of matter. Wiley, New York; 1964. p. 277-83.

[28] Hu X, Van Houte P, Liebeherr M, Walentek A, Seefeldt M, Vandekinderen H. Acta Mater 2006;54:1029-40.

[29] Kurzydlowski KJ, Ralph B. In Proceedings of the 15th Risø international symposium on materials science: numerical predictions of deformation processes and behavior of real materials. Risø, Denmark; 1994. p. 59-74.

[30] Zhang XD, Godfrey A, Huang X, Hansen N, Liu W, Liu Q. In: Proceedings of the 30th Risø international symposium on materials science: nanostructured metals fundamentals to applications. Risø, Denmark: DTU; 2012. p. 409-16.

[31] Danoix F, Julien D, Sauvage X, Copreaux J. Mater Sci Eng 1998;250A:8.

[32] Languillaume J, Kapelski G, Baudelet B. Acta Mater 1997;45:1201.

[33] Gavriljuk VG. Mater Sci Eng 2003;345A:81.

[34] Nam WJ, Bae CM, Oh SJ, Kwon SJ. Scripta Mater 2000;42:457.

[35] Li YJ, Choi P, Borchers C, Westerkamp S, Goto S, Raabe D, Kirchheim R. Acta

Please cite this paper as following

X Zhang, A Godfrey, N Hansen, X Huang. Hierarchical structures in cold-drawn pearlitic steel wire. Acta Materialia 2013;61:4898-4909. 
Mater 2011;59:3965.

[36] Gridnev VN, Gavrilyuk VG, Dekhtyar IY, Meshkov YY, Nizin PS, Prokopenko VG. Phys Stat Sol a 1972;14:689.

[37] Ivanisenko Y, Lojkowski W, Valiev RZ, Fecht HJ. Acta Mater 2003;51:5555.

[38] Sauvage X, Lefebvre W, Genevois C, Ohsaki S, Hono K. Scripta Mater 2009;60:1056.

[39] Wilsdorf HGF, Inal OT, Murr LE. Zeitschrift für Metallkunde 1978;69:701.

Table 1 Summary of wire samples used in this study

\begin{tabular}{|c|c|c|c|c|c|}
\hline Sample & 1 & 2 & 3 & 4 & 5 \\
\hline Diameter $(\mathrm{mm})$ & 1.26 & 0.899 & 0.591 & 0.332 & 0.2 \\
\hline Strain & 0.00 & 0.68 & 1.51 & 2.67 & 3.68 \\
\hline Area reduction (\%) & 0 & 49 & 78 & 93 & 98 \\
\hline
\end{tabular}

Table 2 Thickness of ferrite lamellae (F) and ILS

\begin{tabular}{|c|c|c|c|c|c|}
\hline Strain & 0.00 & 0.68 & 1.51 & 2.67 & 3.68 \\
\hline F $(\mathrm{nm})$ & 70 & 56 & 45 & 23 & 18 \\
\hline ILS (nm) & 89 & 70 & 55 & 28 & 20 \\
\hline
\end{tabular}

Table 3 Area percentage (\%) of deformation microstructure from different initial structures in the longitudinal sections

\begin{tabular}{|c|c|c|}
\hline Strain & Initial A structure & Initial B + C structure \\
\hline 0 & 31.6 & 68.4 \\
\hline & $\begin{array}{c}\text { Deformation microstructure from } \\
\text { initial A structure }\end{array}$ & $\begin{array}{c}\text { Deformation microstructure from } \\
\text { initial B and C structure }\end{array}$ \\
\hline 1.51 & $32.5 \pm 8$ & $67.5 \pm 8$ \\
\hline
\end{tabular}

Table 4 Interlamellar spacing (ILS)

\begin{tabular}{|c|c|c|}
\hline Strain & Structure & ILS (nm) \\
\hline \multirow{3}{*}{0} & A & $95 \pm 28$ \\
\cline { 2 - 3 } & B & $86 \pm 25$ \\
\cline { 2 - 3 } & C & $88 \pm 32$ \\
\hline \multirow{2}{*}{1.51} & A_A & $34 \pm 20$ \\
\cline { 2 - 3 } & A_BC & $65 \pm 30$ \\
\hline 2.67 & A_A & $21 \pm 9$ \\
\hline
\end{tabular}

Please cite this paper as following

X Zhang, A Godfrey, N Hansen, X Huang. Hierarchical structures in cold-drawn pearlitic steel wire. Acta Materialia 2013;61:4898-4909. 


\begin{tabular}{|l|c|c|}
\hline & A_BC & $31 \pm 12$ \\
\hline \multirow{2}{*}{3.68} & A_A & $13 \pm 4$ \\
\cline { 2 - 3 } & A_BC & $23 \pm 14$ \\
\hline
\end{tabular}

\section{Figure captions}

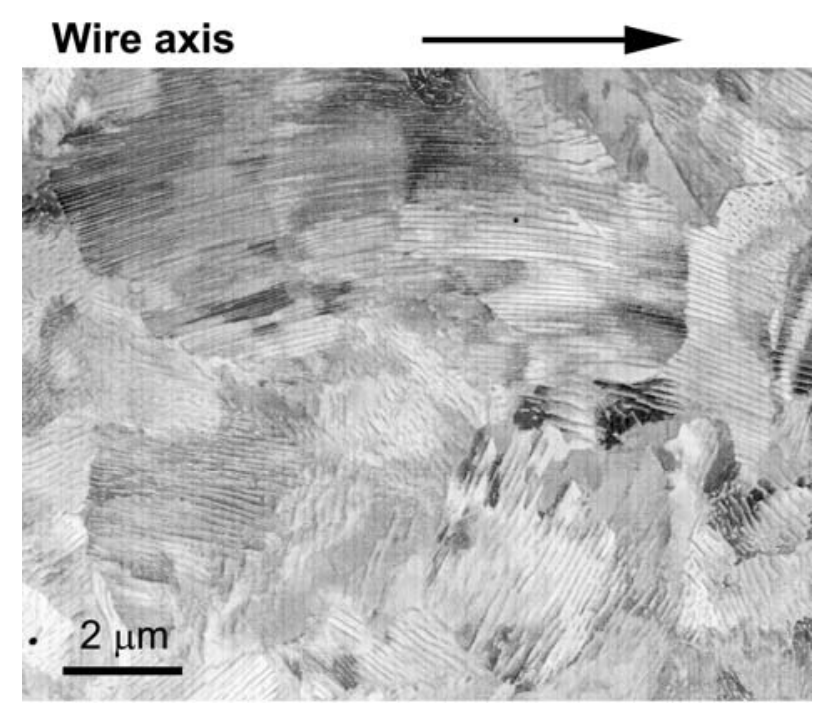

Fig. 1. Backscattered electron image of the lamellar pearlitic microstructure in the initial as-patented wire in the longitudinal section showing no preferred macroscopic orientation of the lamellae with respect to the wire axis (marked).
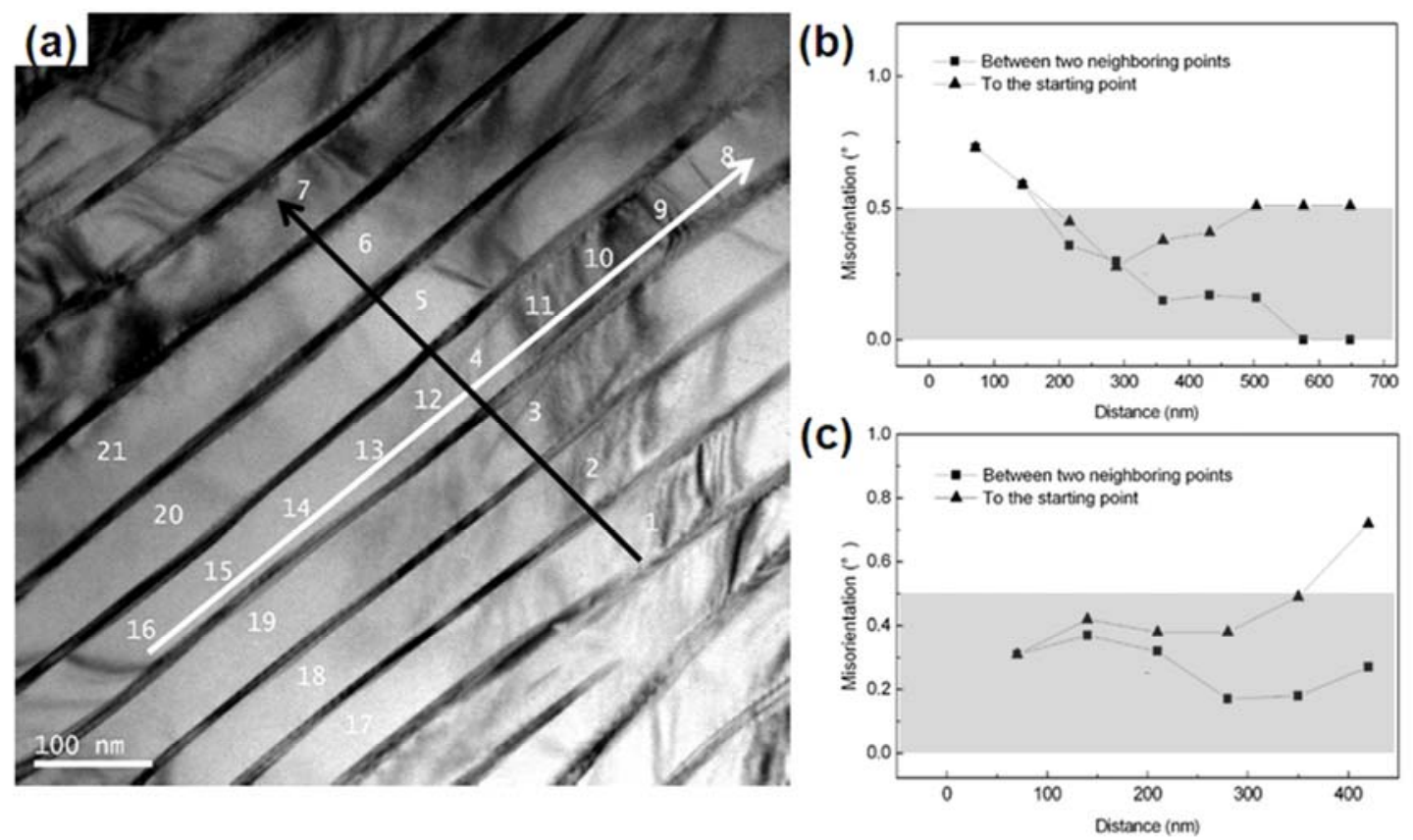

Fig. 2. TEM micrograph of the lamellar structure of pearlite in the patented steel wire

Please cite this paper as following

X Zhang, A Godfrey, N Hansen, X Huang. Hierarchical structures in cold-drawn pearlitic steel wire. Acta Materialia 2013;61:4898-4909. 
(strain 0). (a) TEM micrograph with numbers showing the position of NBD orientation measurements. (b) Graph showing the misorientation angle between neighboring points along the ferrite lamella (white arrow). (c) Graph showing the misorientation angle across the ferrite lamellae (black arrow). The grey shadows in (b) and (c) shows the angular resolution area of misorientation angle measurements.

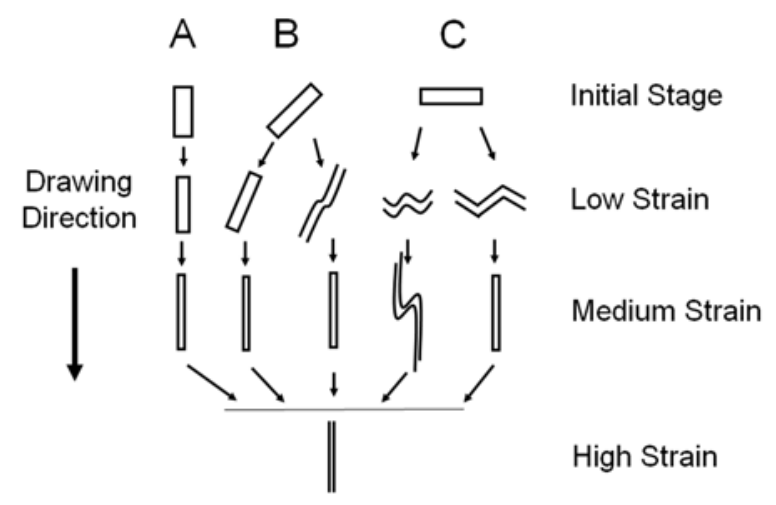

Fig. 3. Schematic illustration of five evolutionary paths for changes in the macroscopic alignment, morphology and thickness of ferrite lamellae in the longitudinal section with cold drawing from low to high strain (see text).

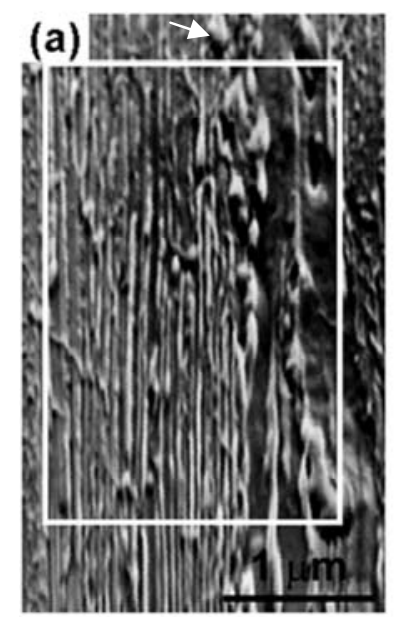

(b)

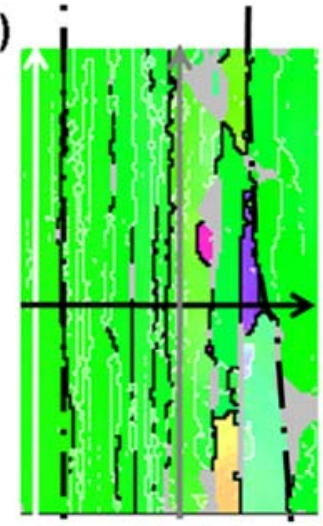

A_A |

(d)

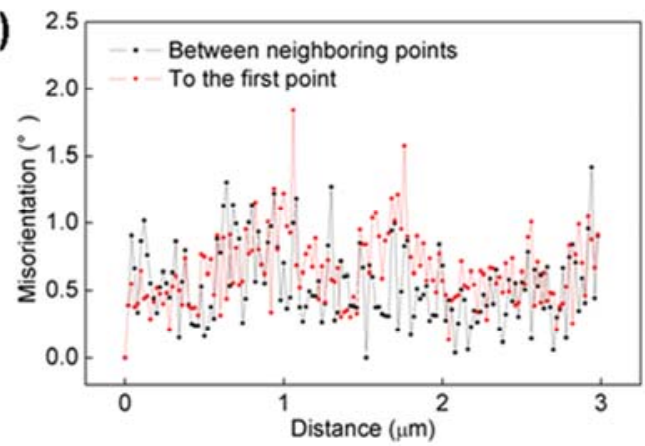

(c)

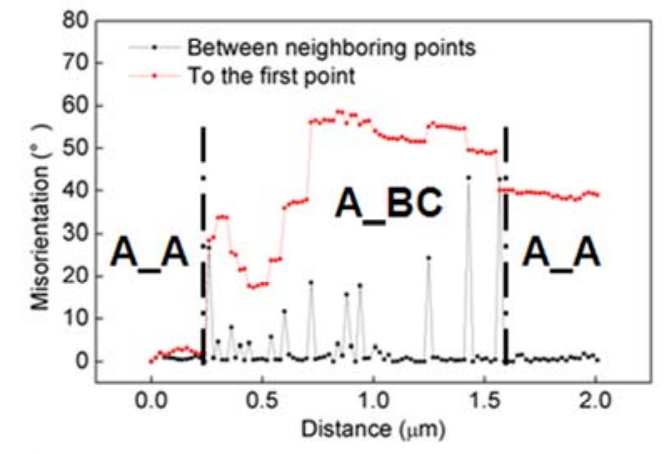

Fig. 4. Image (a) and EBSD map (b) in inverse pole figure (IPF) coloring. The arrows (black, white and grey) mark positions for orientation angle measurements across and along the ferrite lamellae at a strain of 2.67. Angular changes across the lamellae (black arrow) are shown in (c); Angular changes along the lamellae (white and grey

Please cite this paper as following

X Zhang, A Godfrey, N Hansen, X Huang. Hierarchical structures in cold-drawn pearlitic steel wire. Acta Materialia 2013;61:4898-4909. 
arrows) are shown in (d) and (e) for A_A and A_BC structures, respectively. The dashed lines shows the boundaries between A_A and A_BC structure (see text).
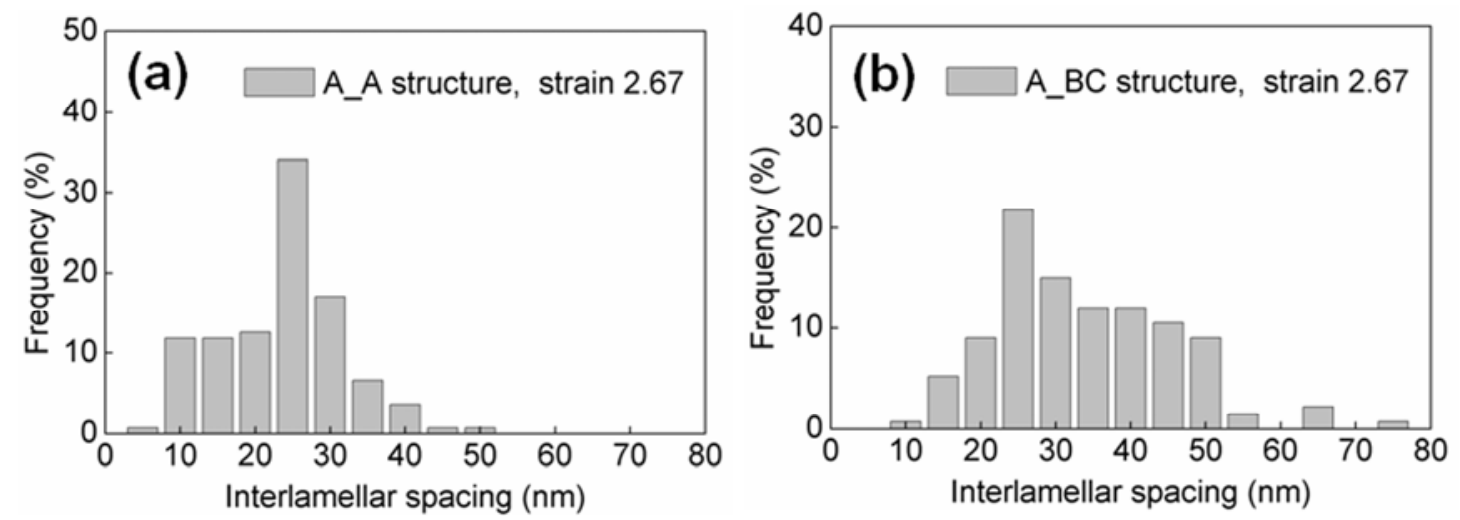

Fig. 5. ILS distribution in A_A (a) and A_BC (b) structure at a strain of 2.67.

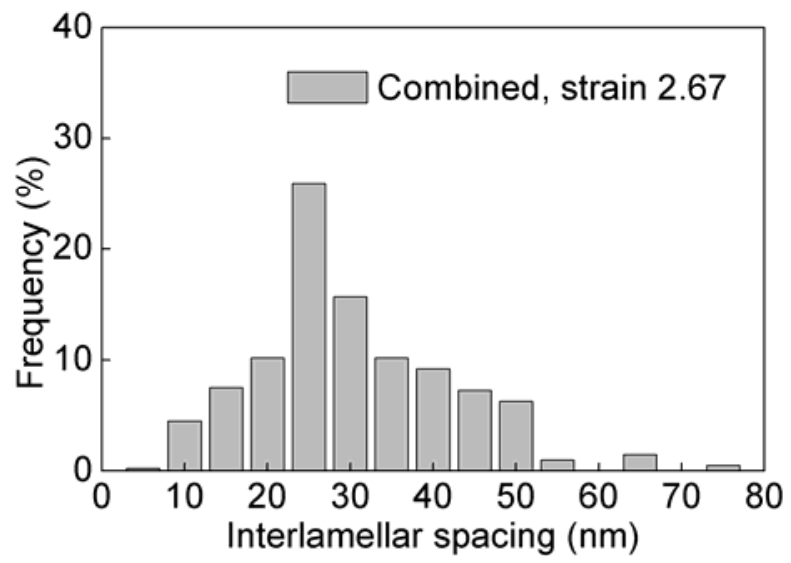

Fig. 6. Combined ILS distribution in A_A and A_BC structures at a strain of 2.67.
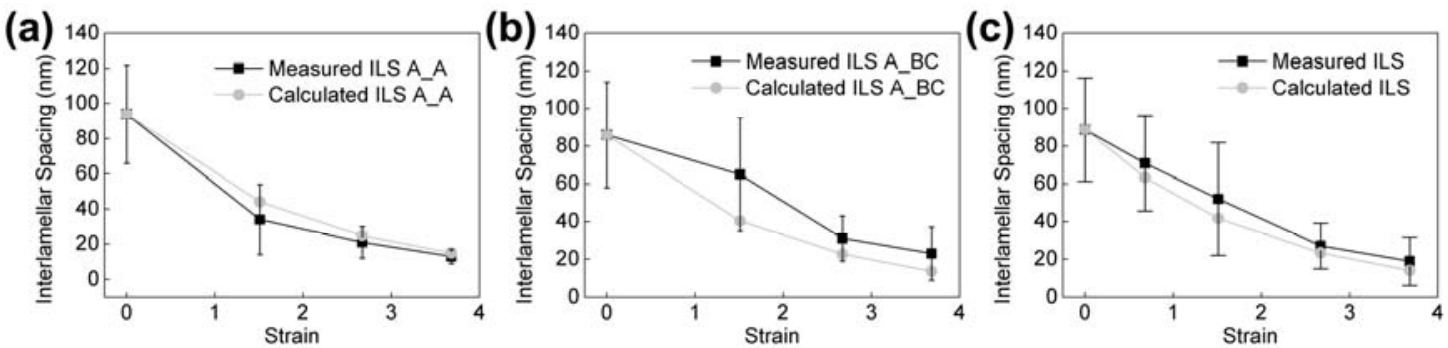

Fig. 7. ILS in A_A (a) and A_BC (b) structure, and combined ILS (c) versus drawing strain. The calculated ILS is based on a $\varepsilon^{-0.5}$ power law relationship.

Please cite this paper as following

X Zhang, A Godfrey, N Hansen, X Huang. Hierarchical structures in cold-drawn pearlitic steel wire. Acta Materialia 2013;61:4898-4909. 

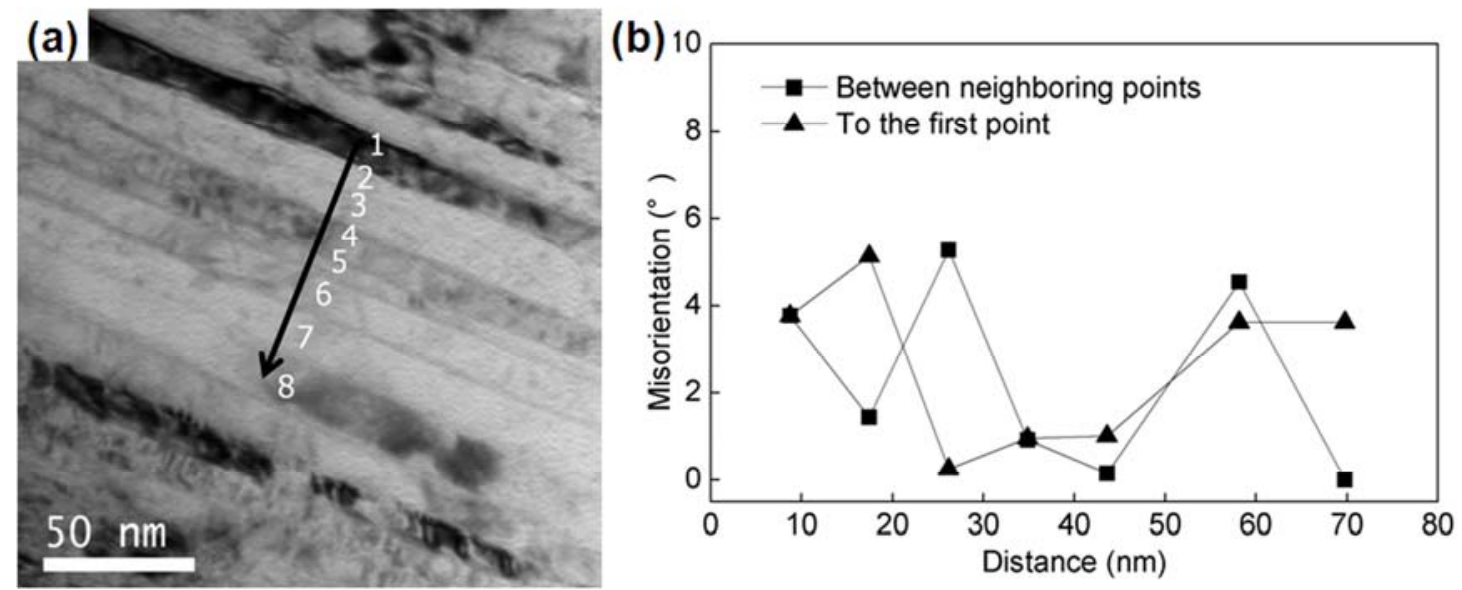

Fig. 8. (a) TEM micrograph with numbers showing the positions of nanobeam diffraction orientation measurements and (b) graph showing the misorientation between neighboring ferrite lamellae of A_A sructure at a strain of 3.68.
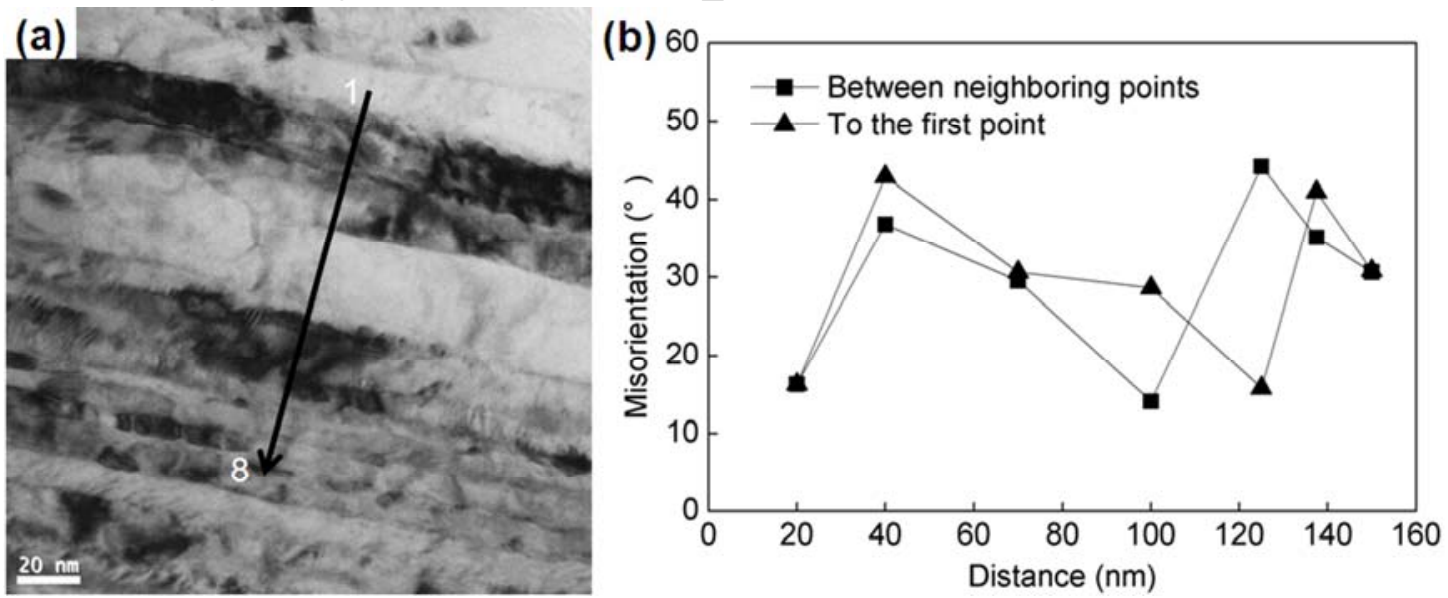

Fig. 9. (a) TEM micrograph with numbers showing the positions of nanobeam diffraction orientation measurements and (b) graph showing the misorientation angle across ferrite lamellae in an A_BC sructure at a strain of 3.68 .

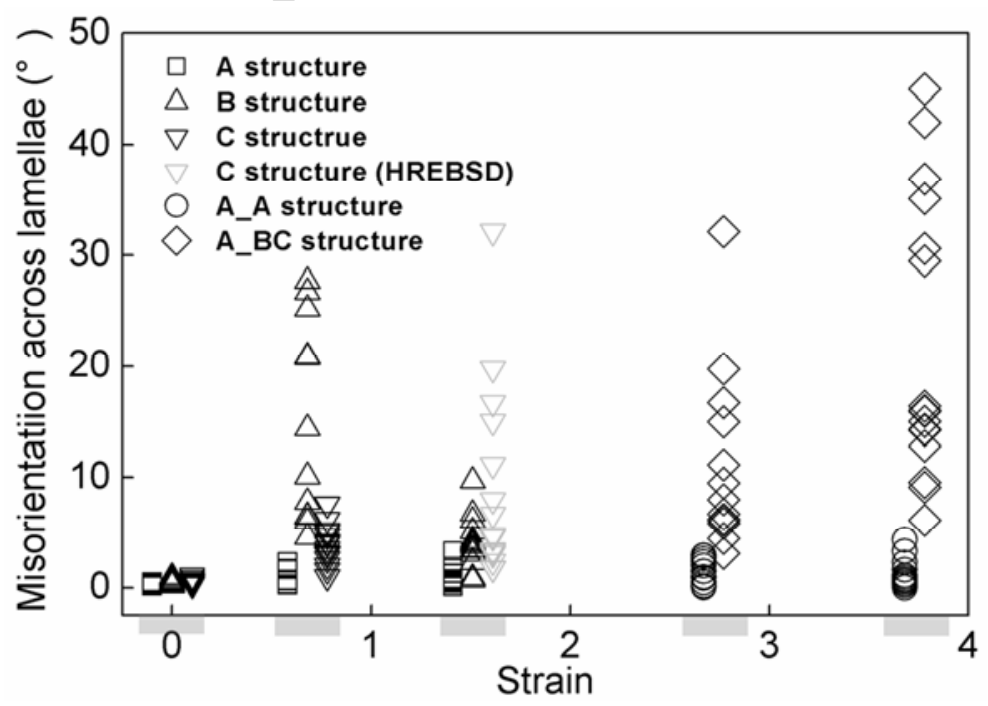

Fig. 10. Graph showing misorientation angles across the ferrite lamellae determined

Please cite this paper as following

X Zhang, A Godfrey, N Hansen, X Huang. Hierarchical structures in cold-drawn pearlitic steel wire. Acta Materialia 2013;61:4898-4909. 
by HREBSD and TEM versus the strain in structures with different deformation paths. The light grey bars at the strain axis represent the five strains in this study in order to show the differences and data spread in misorientation angle as the drawing strain increases from low to high value. Note that this figure also contains data for the A, B and $\mathrm{C}$ structures at the strains of 0 and 0.68 .
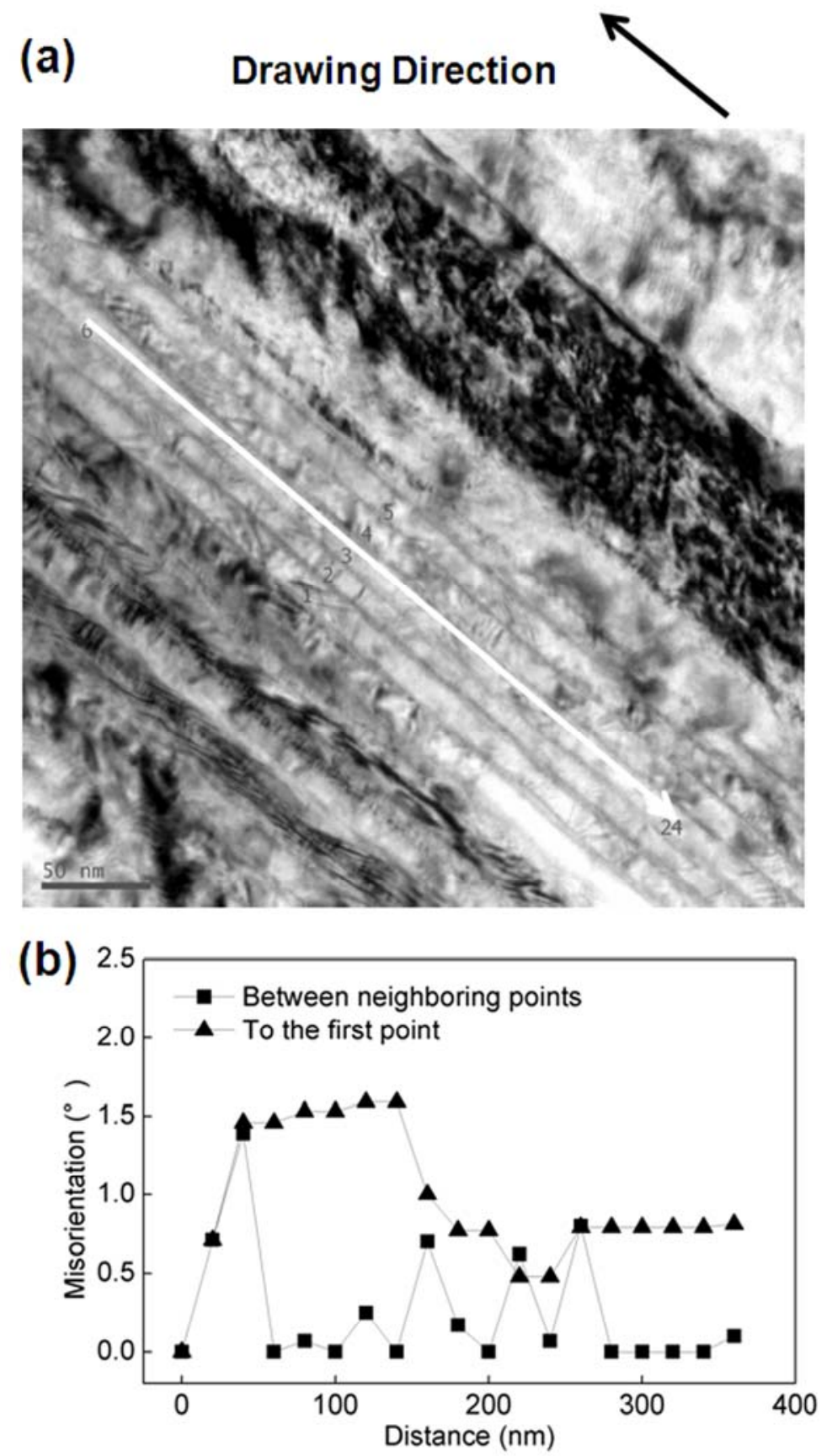

Fig. 11. (a) TEM micrograph and (b) orientation change along a ferrite lamellae (white arrow) in an A_A structure at a strain of 3.68.

Please cite this paper as following

X Zhang, A Godfrey, N Hansen, X Huang. Hierarchical structures in cold-drawn pearlitic steel wire. Acta Materialia 2013;61:4898-4909. 

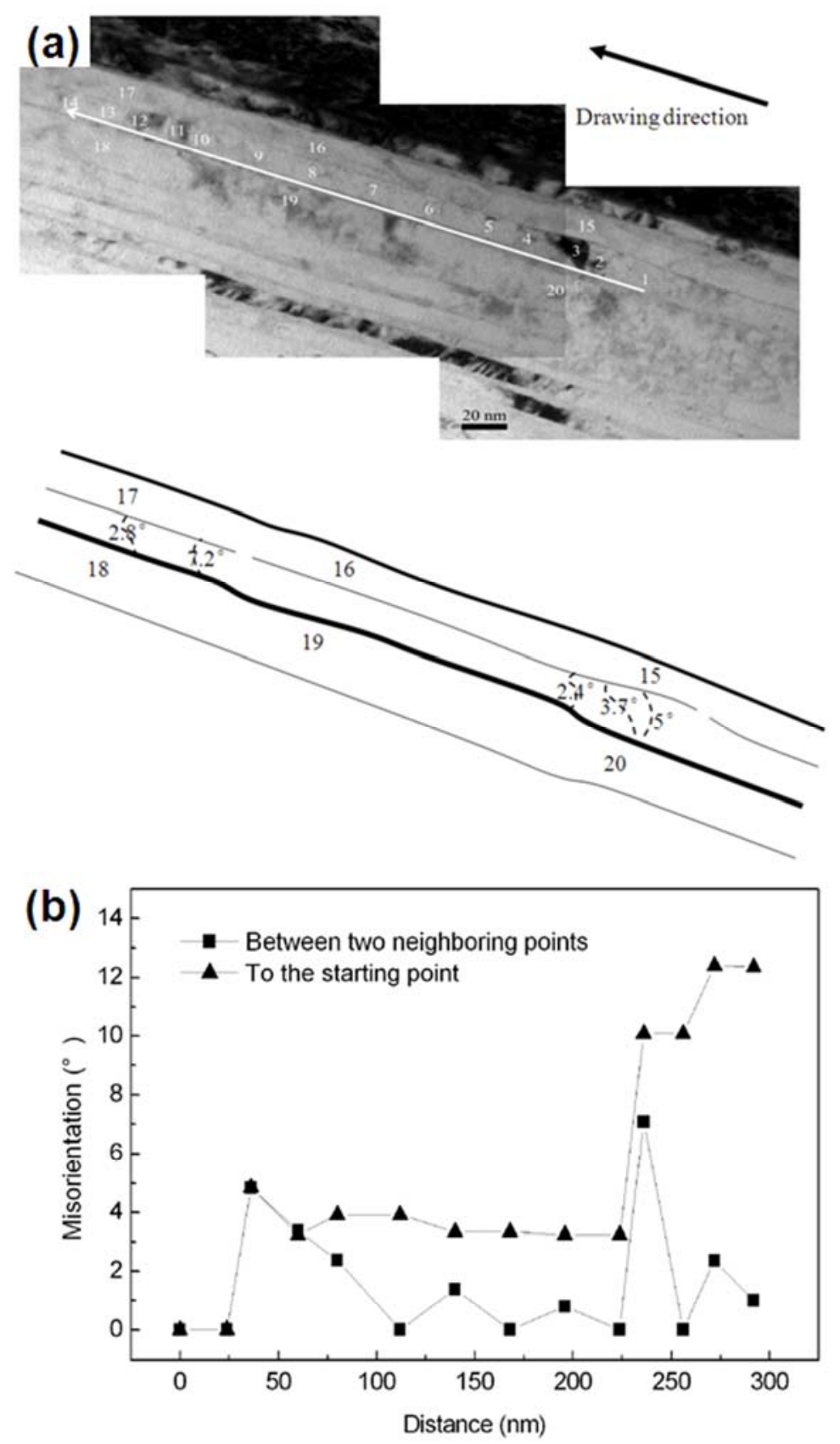

Fig. 12. (a) TEM micrograph and sketch with solid lines representing cementite lamellae and dashed lines in the ferrite lamellae showing LABs and (b) orientation change along a ferrite lamella (white arrow) in an A_BC structure at a strain of 3.68.

Please cite this paper as following X Zhang, A Godfrey, N Hansen, X Huang. Hierarchical structures in cold-drawn pearlitic steel wire. Acta Materialia 2013;61:4898-4909. 


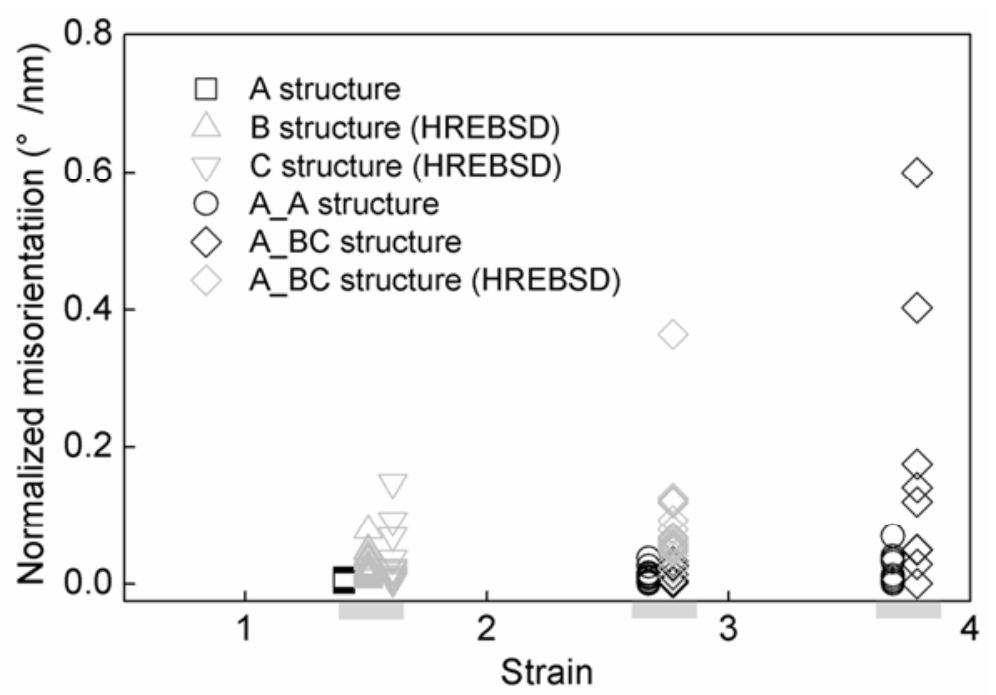

Fig. 13. Normalized misorientation angles along the ferrite lamellae versus strain in structures with different deformation paths. The light grey bar at the strain axis represent the three strains in this study in order to show the differences and data spread in the misorientation across the ferrite lamellae related to the initial structure.

Please cite this paper as following

X Zhang, A Godfrey, N Hansen, X Huang. Hierarchical structures in cold-drawn pearlitic steel wire. Acta Materialia 2013;61:4898-4909. 